\title{
Relación entre desempeño corporativo y estructura de propiedad en Chile*
}

\author{
Zuniga-Jara, Sergio** \\ Soria, Karla***
}

\section{Resumen}

En este estudio se analiza la relación entre la concentración de la propiedad y el desempeño corporativo para las principales sociedades anónimas abiertas de Chile, en el periodo 2003-2008. Se trata de una investigación aplicada de tipo cuantitativa y explicativa. Los resultados de las regresiones muestran que cuando se considera como indicador de desempeño el rendimiento de mercado del patrimonio, la relación concentración-desempeño es virtualmente inexistente en Chile. También se verifica que, como se esperaba, el coeficiente de riesgo sistemático logra explicar fuertemente el desempeño. Por otro lado, ninguna de las variables explicativas utilizadas logra afectar los rendimientos de mercado de las empresas. Es más, tampoco logran afectar la relación desempeño-concentración de la propiedad. Como conclusión, ninguna de las características de la empresa, y ninguna de las características de los principales propietarios logra derrotar al riesgo sistemático en su poder explicativo de los retornos. Además, tampoco contribuyen estadísticamente a su explicación.

Palabras clave: Desempeño corporativo, concentración, gobierno corporativo, estructura de propiedad.

\section{Relation between Corporate Performance and Property Structure in Chile}

\section{Abstract}

This study analyzes the relationship between the concentration of property and corporate performance for the main, open anonymous societies in Chile during the 2003-2008 period. The research is applied and of the quantitative, explanatory type. Results of the regressions show that when the

\section{Recibido: 09-06-11. Aceptado: 08-02-13}

* Los autores agradecen el apoyo financiero del Núcleo de la Iniciativa Científica Milenio "Ciencia Regional y Políticas Públicas" (MIDEPLAN, Chile).

** Doctor en Finanzas, Magíster en Finanzas. Académico de la Escuela de Ingeniería Comercial, Universidad Católica del Norte, Chile, e-mail: sz@ucn.cl

*** Doctor en Administración, Magíster en Finanzas. Académico de la Escuela de Ingeniería Comercial, Universidad Católica del Norte, Chile, e-mail: ksoria@ucn.cl 
market performance of assets is considered as a performance indicator, the relationship of concentration to performance is virtually non-existent in Chile. Also, the results verify that, as expected, the systematic risk coefficient â has a strong effect on explaining performance. On the other hand, none of the explanatory variables utilized affect the market performances of the companies, nor do they affect the performance-concentration relationship of the property. In conclusion, none of the characteristics of the company and none of the characteristics of the principle owners are able to defeat systematic risk and its explanatory power over returns. Furthermore, they do not contribute statistically to its explanation.

Keywords: Corporate performance, concentration, corporate government, property structure.

\section{Introducción}

La estructura de propiedad de las empresas, ya sea en términos de la concentración de su propiedad o de las características de los propietarios, parece tener implicancias en el desempeño de las mismas. En su trabajo pionero, Berle y Means (1932) destacaron que las leyes corporativas y tributarias han empujado a la separación de la propiedad y del control, llevando a una mayor dispersión de la propiedad. Esto a su vez ha llevado a que los pequeños accionistas estén menos interesados en la gestión, y los que están realmente involucrados (gerentes y directores) pueden obtener ventajas personales, debido a la falta de un control efectivo de los propietarios-accionistas, con la consiguiente pérdida patrimonial. Becht et al. (2003) proveen una extensa revisión de estudios a nivel internacional que regresionan medidas de desempeño con medidas de control corporativo.

Jensen y Meckling (1976) han señalado que una mayor propiedad accionaria en manos de los ejecutivos de las empresas implica una estructura de incentivos más alineada con los intereses de los accionistas. Así, una forma de reducir los costos de agencia y mejorar el desempeño parece encontrarse en la concentración de la propiedad. En efecto, existe evidencia de que el desempeño de los administradores aumenta cuando hay mayor concentración, pues los grandes accionistas tienen fuertes incentivos para monitorear eficientemente (Shleifer y Vishny, 1997). La identidad de los grandes propietarios (familias, bancos, gobierno, entre otros) también parece tener implicancias relevantes en la estrategia corporativa y en el desempeño. Al respecto, Thomsen y $\mathrm{Pe}$ derson (2000) encuentran que los inversionistas financieros pueden ser asociados con mayor rentabilidad y valor para los accionistas, aunque con menores crecimientos en las ventas.

La existencia de inversionistas con altos incentivos para aumentar el desempeño también parece traducirse en la capacidad de realizar más a menudo reestructuraciones. Esto sugiere que muchos administradores hicieron las reestructuraciones presionados por los grandes accionistas (Bethel y Liebeskind, 1993). También, la desinversión parece estar directamente relacionada con el desempeño de las empresas, lo que está precedido por un gobierno débil al interior de las mismas (Hoskisson et al., 1994).

De acuerdo a Brailsford et al. (2002), la concentración de la propiedad también parece tener un efecto significa- 
tivo en el nivel de endeudamiento, es decir en la forma en que se distribuye la propiedad entre administradores y grupos de accionistas. También se ha reportado diferencias significativas entre concentración y desempeño a través de los países, de modo que el sistema nacional de gobierno corporativo parece ser también relevante. Gedajlovic y Shapiro (1998) argumentan que el impacto de la concentración de propiedad es más débil en Francia, donde los principales propietarios son bancos, corporaciones no financieras y el Gobierno. En Japón, Morck et al. (2000) encuentran que los $Q$ ratios caen al aumentar la proporción de propiedad de los grandes bancos. También señalan que en Japón, a diferencia de los EEUU, el valor de las empresas aumenta monótonamente con el porcentaje de propiedad por parte de los administradores. Orr et al. (2005) reportan que para Nueva Zelandia la composición de la gerencia de empresas con altas opciones de crecimiento está positivamente relacionada al valor de las empresas.

En Latinoamérica, la fuerte concentración de la propiedad parece ser una característica de las empresas. De acuerdo a Fuenzalida et al. (2008), en el mundo los tres principales accionistas concentran cerca del $46 \%$ de la propiedad, mientras que en Suramérica este porcentaje se elevaría a un $70 \%$. Los mismos autores, para un panel de empresas de cinco mercados suramerica- nos, obtienen que las empresas con mayor concentración generan rendimientos superiores. Otros estudios similares para Latinoamérica incluyen a Argentina (De Michele, 2000; Apreda, 2001 y Bebczuk, 2005), México (Castañeda, 2000; Husted y Serrano, 2002) y Colombia (Gutiérrez et al., 2008).

En Chile se ha hecho limitada investigación empírica en este campo; destacan los trabajos de Le Fort y Walker (1999) quienes reportan que los controladores de los conglomerados chilenos mantienen más capital del necesario para tener el control de las empresas, lo que se asocia a la existencia de beneficios en la mantención de acciones de las empresas subsidiarias. Agosin y Pastén (2003) señalan que en Chile el sistema de control tiene un nivel de discriminación moderado contra pequeños accionistas y tenedores de bonos, esto debido al monitoreo de las AFP y la regulación. Maquieira et al. (2003) estudian las compañías de inversión en Chile encontrando un castigo promedio de $28 \%$ debido al mayor control corporativo, debido a que los mayoritarios maximizan su bienestar en desmedro de los minoritarios. Espinoza y Maquieira (2010) no encuentran una relación significativa entre estructura de propiedad y desempeño para empresas chilenas emisoras de ADR ${ }^{1}$.

Basados en la discusión anterior, este estudio se centra en analizar la relación entre desempeño y concentración de la propiedad para las empresas chile-

1 Un American Depositary Receipt (ADR), es un título que respalda el depósito en un banco estadounidense de acciones de compañías constituidas fuera de aquel país, de manera de poder transar las acciones de esa compañía en los EEUU. 
nas. La principal hipótesis de trabajo es que el desempeño corporativo mejora a medida que el nivel de concentración de la propiedad aumenta, adicionalmente se analiza si esta posible relación se mantiene cuando se controla por variables estructurales, incluyendo diferentes medidas de riesgo de las empresas, las características de la empresa, y las características del accionista mayoritario.

Se reportan estimaciones de concentración de la propiedad para las empresas en estudio, y se justifica y estima una medida de desempeño corporativo. Posteriormente, se estima la relación desempeño-concentración de las empresas, para finalmente construir variables que caracterizan tanto al riesgo de las empresas, a los propietarios principales como a las empresas. Estas medidas son incluidas como variables de control en el análisis de la relación desempeño-concentración.

Para la construcción de la base de datos se identificó las sociedades que realizaron transacciones en la Bolsa de Comercio de Santiago de Chile (BCS) durante los años 2008 y 2004, debido a que éstos fueron los últimos cuatro años disponibles al momento de realizar el estudio. Esto arrojó inicialmente un total de 146 empresas. Los Estados Financieros (EEFF) de estas empresas fueron recopilados a partir de la base de datos de la Superintendencia de Valores y Seguros de Chile (SVS) y de la Superintendencia de Bancos e Instituciones Financieras (SBIF) para dichos años. Se adoptó el criterio de seleccionar Estados Financieros (EEFF) consolidados, y a falta de estos usar EEFF individuales. En el caso de las sociedades con más de una serie de acciones en circulación, su patrimonio bursátil se obtuvo de la suma de los patrimonios de cada serie. En estas empresas, para la estimación de la volatilidad de precios se seleccionó la serie de acciones con mayor presencia bursátil.

Se realizó un proceso de depuración de la base de datos, que incluyó la eliminación de las empresas que habiendo transado en la BCS durante 2008 , sus estados financieros no aparecen en la SVS. Se identificaron y eliminaron las empresas que no reportaron ventas en los años 2004 y 2008 (o reportaron ventas en solo alguno de esos años), y las empresas con muy baja presencia en Bolsa. De igual forma se eliminaron algunos casos muy aislados de empresas con 'datos extraños' en sus EEFF, y que hubiesen requerido un análisis detallado para encontrar una explicación (por ejemplo, empresas que reportaron en el periodo una variación en su patrimonio mayor a $1000 \%$, empresas con patrimonio negativo, empresas que pagan el $3500 \%$ de sus utilidades como dividendos, empresas con patrimonio negativo). Por simplicidad, todos estos casos fueron considerados outliers. Con esto, la base de datos quedó compuesta por 112 empresas.

Puesto que la mayoría de los casos las sociedades que transan en la BCS constituyen la matriz de un grupo de empresas, las que desarrollan varios giros simultáneamente, resulta poco preciso clasificarlas en un grupo o sector industrial específico, por lo que se omite un análisis de este tipo. 


\section{Concentración de la propiedad}

La concentración de la propiedad de una empresa es usualmente medida como el porcentaje de la propiedad que pertenece al principal accionista, es decir al accionista mayoritario. La evidencia de los efectos de una alta concentración de propiedad es mixta. Si bien parece existir una relación positiva entre mayor concentración de la propiedad y mejor desempeño (y rentabilidad), esta relación también parece perderse para muy grandes accionistas (Thomsen y Pederson, 2000). Algunos estudios (Morck et al., 1988; McConnell y Servaes, 1990) sugieren que esta relación es no-lineal, de forma tal que a bajos niveles de concentración, los costos de monitoreo superan los beneficios, y a muy altos niveles de concentración los administradores son presionados en exceso. Acharya y Bisin (2009) desarrollan un modelo para dar una explicación estructural de la relación no lineal entre el desempeño (medido por el ratio $Q$ de Tobin) y la propiedad de las empresas.

En este estudio se define la concentración de la propiedad como el porcentaje de propiedad del principal accionista o accionista mayoritario. Los resultados de las estimaciones de concentración para las 112 empresas consideradas en este estudio se muestran en la Tabla 1. Esta tabla clasifica las empresas en estudio en tres grupos, de acuerdo a la proporción de la propiedad que mantiene en ellas el principal accionista. Así, por ejemplo, en el grupo de alta concentración (es decir más de un $33,33 \%$ de la propiedad de las empresas pertenece a un solo accionista) hay $14 \mathrm{em}$ presas, y el porcentaje medio de propiedad que mantiene en ellas el principal accionista es de $79.5 \%$, y de este mismo modo se interpretan los otros dos grupos. En la tabla se puede apreciar que la concentración en Chile en el periodo estudiado tiene una media de $43.3 \%$, lo que en principio no puede considerarse extremadamente alto en comparación al estándar internacional, considerando que a través de un cálculo idéntico Thomsen y Pedersen (2000: 547) reportan para Europa un 39\% en promedio, y Gedajlovic y Shapiro (1998) reportan para EEUU un promedio de 19\%, 21\% para el Reino Unido, y $68.5 \%$ para Alemania. Para varios países latinoamericanos, Fuenzalida et al. (2008) reportan concentraciones entre $44 \%$ (Colombia) y $61 \%$ (Argentina). Para una muestra reducida, Majluf et al. (1988) reportan estimaciones similares en Chile (40\%).

Tabla 1

Concentración de la Propiedad (2003-2008)

\begin{tabular}{lcc}
\hline & Número de empresas & $\%$ de la propiedad del mayor accionista \\
\hline Concentración Alta & 14 & $79,5 \%$ \\
Concentración Media & 58 & $49,1 \%$ \\
Concentración Baja & 40 & $22,3 \%$ \\
Total & 112 & $43,3 \%$ \\
\hline
\end{tabular}

Fuente: Elaboración propia. 
A continuación se clasifican las empresas bajo estudio en base a la concentración de su propiedad, considerando: como concentración baja si ésta es < 1/3; concentración alta si ésta es $>2 / 3$, y concentración media en otro caso. De acuerdo a esto, la segunda columna de la Tabla 1 muestra una distribución sesgada hacia la izquierda, es decir un mayor número de empresas con baja concentración (40 empresas) respecto a los casos de alta concentración (14 empresas). El Gráfico 1 ilustra este punto, mostrando la distribución de frecuencias para la concentración de la propiedad de las empresas en estudio.

\section{Desempeño corporativo}

En la medición del desempeño de las empresas, coexisten básicamente dos enfoques. Las medidas contables ${ }^{2}$ generalmente utilizan la rentabilidad sobre los activos (ROA) y la rentabilidad sobre el patrimonio (ROE) como principales indicadores. Las medidas económico-financieras por otra parte, utilizan medidas corregidas por riesgo entre las que destacan las de Jensen (1968) y Treynor (1966). La argumentación de estas últimas subyace en que las medidas basadas en datos contables tienen una serie de limitaciones, dentro de las que destacan especialmente el hecho de que se basan en completamente en los Estados Financieros de las empresas, los que a su vez no reflejan necesariamente los valores de mercado, y además están sujetos a los criterios sobre los cuales se construyen los mismos sistemas contables. En los años recientes, cada vez toma mayor importancia la medición del desempeño de las empresas basadas en medidas de

\section{Gráfico 1}

Proporción de la propiedad mantenida por el mayor accionista 2003-2008

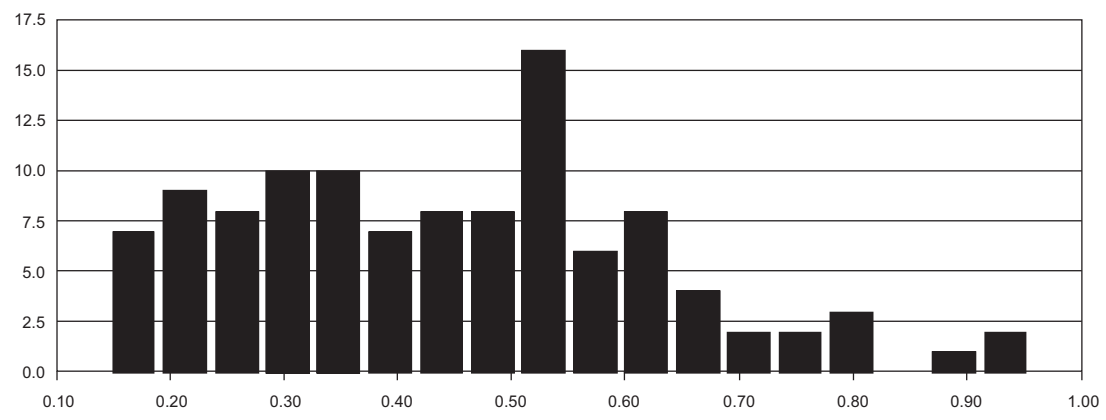

Fuente: Elaboración propia.

2 Algunas de las más usadas son la rentabilidad sobre los activos (ROA), la rentabilidad sobre el patrimonio (ROE), el rendimiento por dividendos, y la relación patrimonio de mercado a patrimonio a valor libro (ratio $Q$ de Tobin). 
retorno efectivo para los propietarios, a través de medidas de creación de valor a precios de mercado.

En Chile parecen existir importantes distorsiones en la información contable reconocidas por la autoridad ${ }^{3}$, por lo que el uso de medidas de desempeño contable resta validez a las mismas como fuente de información económica, y limita su comparabilidad con la de otros países. Solo a partir de 2009 se implementa obligatoriamente en Chile la norma International Financial Reporting Standards (IFRS) para las sociedades inscritas en la SVS, lo que debe traducirse en importantes mejoras futuras en la calidad de la información contenida en los EEFF.

Por este motivo, en este estudio se emplea una medida de desempeño basada en el rendimiento de mercado, medido por las variaciones de precios accionarios ajustados por variaciones de capital. Entre los autores que han asumido esta medida se puede citar a Murali y Welch (1989). La ecuación de rendimiento continuo usada viene dada por:

$$
R_{t} \log \frac{P_{t}}{P_{t-1}}
$$

(Ecuación 1)

donde $P_{t}$ es el precio de cierre en el mes t para las acciones de una sociedad determinada, $R_{t}$ es la rentabilidad mensual del título, y $\log ($ ) es el logaritmo natural. En todos los casos se usa una serie de precios mensual para el periodo entre 2004 y 2008. El Gráfico 2 muestra el histograma para esta serie, y es construido en base a los rendimientos mensuales promedio de las 112 sociedades consideradas en el estudio para el periodo 2003-2008. Aquí

Gráfico 2

Retornos accionarios mensuales 2003-2008

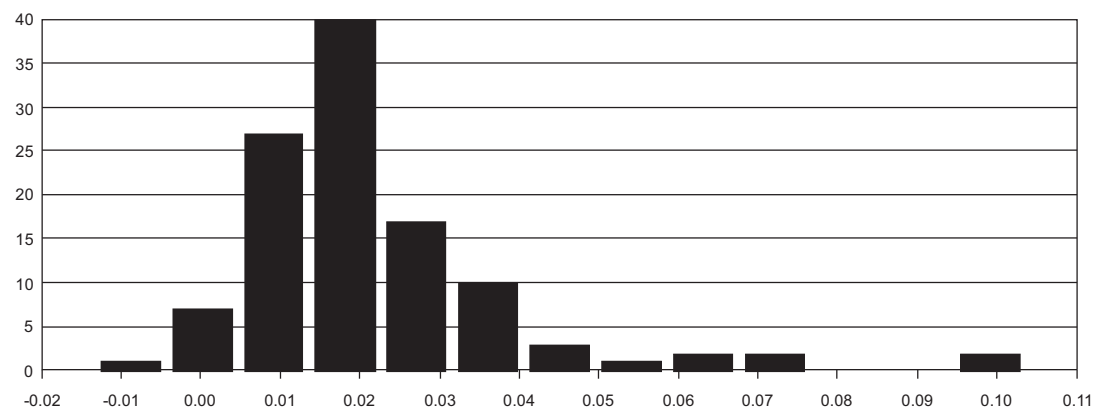

Fuente: Elaboración propia.

3 El Superintendente de Valores (SVS) de Chile, Alejandro Ferreiro, señaló que “...más del 50\% de la normativa contable chilena es diferente a la internacional (...) Estamos bastante desalineados" (Diario Financiero, 28/11/2005). A raíz de esto, a partir de 2009 comenzó un proceso de adopción a las IFRS (International Financial Reporting Standards) o Normas Internacionales de Información Financiera, para lograr que los balances chilenos sean comparables a los de empresas de más de 100 países. 
se reporta el característico sesgo a la izquierda, a pesar de que las pruebas estadísticas no permitieron confirmar que en la mayoría de los casos éstos retornos se distribuyen lognormal.

\section{Influencia de la concentración de la propiedad en el desempeño}

El objetivo principal de este estudio es analizar el efecto que existe entre la concentración de la propiedad de las empresas por sobre su desempeño en Chile. Los resultados obtenidos hasta ahora son mixtos, basados en los reportes de Agosin y Pastén (2003), Maquieira et al. (2003) y Espinoza y Maquieira (2010).

Se realizan las estimaciones, utilizando para ello un modelo de regresión lineal. En este modelo se postula que la rentabilidad, es decir el desempeño de las empresas, depende linealmente de una serie de variables explicativas. Las primera de ellas es justamente la estructura de propiedad, y las restantes son una serie de diferentes variables de control, las que tienen por objeto neutralizar sus efectos por sobre la variable dependiente, $y$ de esa forma poder medir con mayor precisión la relación entre la variable independiente de interés y la variable dependiente. Si no se incluyeran variables de control en las mediciones, entonces la relación que se mediría entre desempeño-propiedad estaría contaminada por dichas variables, las que efectivamente están también afectando el desempeño, pero al no estar explicitadas en el modelo, todos estos efectos sería capturados por la única variable independiente que se use. Las variables de control capturan entonces las interacciones omitidas, y distintas a la interacción analizada.

El modelo utilizado en este estudio para capturar la relación entre el desempeño de la empresa $\left(R_{i}\right)$ y la propiedad de los accionistas $\left(C_{i}\right)$, es el siguiente:

$R_{i} \quad 0_{1} C_{i} \quad{ }_{j}^{n} i_{1}{ }_{i} 0_{j i} \quad$ (Ecuación 2)

donde $C_{i}$ es la medida de concentración de la propiedad de la empresa i-ésima, $O_{j i}$ se refiere a cada una de las j-ésimas variables de control, $y,{ }_{i}$ al término de error, respectivamente.

La estructura de la base de datos usada es fundamentalmente un corte transversal de datos para 2008, aunque algunas variables son construidas en base a una serie de tiempo. En este sentido seguimos el enfoque de Thomsen y Pedersen (2000), quienes trabajan con datos del año 1990 solamente.

La Tabla 2 muestra las estimaciones de la Ecuación 2; reporta los resultados de regresionar los rendimientos accionarios de las 112 empresas del estudio, usando distintos regresores. Se consideran siete (7) modelos a saber: El modelo 1 incluye solamente una medida de la concentración de la propiedad. Los modelos 2 y 3 incorporan dos medidas alternativas de riesgo sistemático de las empresas. Los modelos 4 y 5 incluyen dos características de las empresas: presencia en bolsa y AFPabilidad. Finalmente, los modelos 6 y 7 agregan dos características de los principales accionistas de las empresas: si éste se encuentra inscrito en la SVS (lo que lo obliga a reportar sus estados financieros y otra información relevante), y el hecho de que éste 
Tabla 2

Regresiones para la relación desempeño - concentración 2003-2008

\begin{tabular}{|c|c|c|c|c|c|c|c|}
\hline & Modelo 1 & Modelo 2 & Modelo 3 & Modelo 4 & Modelo 5 & Modelo 6 & Modelo 7 \\
\hline Constant & $\begin{array}{c}0.0208\left(^{\left({ }^{* *}\right)}\right. \\
(4.397)\end{array}$ & $\begin{array}{c}0.0095\left(^{* * *}\right) \\
(1.976)\end{array}$ & $\begin{array}{l}0.0019 \\
(0.694)\end{array}$ & $\begin{array}{l}0.0179 \\
(4.173)\end{array}$ & $\begin{array}{c}0.0237\left(^{* * *}\right) \\
(3.800)\end{array}$ & $\begin{array}{c}0.0204\left({ }^{* * *}\right) \\
(4.442)\end{array}$ & $\begin{array}{c}0.0212\left(^{* * *}\right) \\
(4.406)\end{array}$ \\
\hline Concentration & $\begin{array}{l}-0.0035 \\
(-0.364)\end{array}$ & $\begin{array}{l}0.0008 \\
(0.109)\end{array}$ & $\begin{array}{l}-0.0034 \\
(-0.584)\end{array}$ & $\begin{array}{l}-0.0022 \\
(-0.243)\end{array}$ & $\begin{array}{l}-0.0025 \\
(-0.262)\end{array}$ & $\begin{array}{l}-0.0049 \\
(-0.489)\end{array}$ & $\begin{array}{l}-0.0035 \\
(-0.367)\end{array}$ \\
\hline Beta_Local & & $\begin{array}{c}0.01519\left(^{* * *}\right) \\
(3.613)\end{array}$ & & & & & \\
\hline Beta_Ajust & & & $\begin{array}{c}0.0091\left(^{* * \star}\right) \\
(16.392)\end{array}$ & & & & \\
\hline Presencia & & & & $\begin{array}{l}0.0000 \\
(1.094)\end{array}$ & & & \\
\hline Afpeabilidad & & & & & $\begin{array}{l}-0.0045 \\
(-0.850)\end{array}$ & & \\
\hline PPAL_ACC_SVS & & & & & & $\begin{array}{l}0.0030 \\
(0.630)\end{array}$ & \\
\hline PPAL_ACC_SA & & & & & & & $\begin{array}{l}-0.0006 \\
(-0.173)\end{array}$ \\
\hline Num.Obs. & 112 & 112 & 112 & 112 & 112 & 112 & 112 \\
\hline Hausman & & $\left.36.1198{ }^{(* * *}\right)$ & $\left.215.8610^{* * *}\right)$ & 1.0966 & 1.1515 & 0.5433 & 0.0128 \\
\hline Hausm. Signif. & & 0.0000 & 0.0000 & 0.2973 & 0.2856 & 0.4626 & 0.9101 \\
\hline R2 Ajust & -0.00779 & 0.2361 & 0.6588 & -0.0069 & -0.0064 & -0.0120 & -0.0169 \\
\hline
\end{tabular}

$\left.{ }^{*}\right)$ signif. al $10 \% ;\left(^{* *}\right)$ signif. al $\left.5 \% ;{ }^{* * *}\right)$ signif. al $1 \%$

Fuente: Elaboración propia.

está organizado legalmente como una sociedad anónima o no. Debajo de cada coeficiente se reporta el estadístico t de student.

Todos los errores estándar de los coeficientes de las regresiones provienen de estimaciones consistentes por heteroscedasticidad de White (1980). Se reporta también el estadístico de Hausman

(1978) y su significancia, para evaluar la endogeneidad de las variables de control. Para evaluar y corregir la robustez de los resultados se usa el procedimiento de White (1980), obteniendo estimaciones de errores estándar y covarianzas consistentes respecto a heteroscedasticidad. Correcciones por causalidad inversa no han sido realizadas ${ }^{4}$.

4 El procedimiento estándar de diagnosticar y corregir el problema de autoselección (causalidad inversa) es el enfoque de dos pasos de Heckman (1979). La pertinencia de este análisis es clara en el caso de modelos con variable explicativa dicotómica, o en casos en los cuales la variable explicativa está compuesta por dos grupos, uno de los cuales está sub-representado por los datos. Sin embargo, en nuestro caso, tanto el modelo básico como las variables no se ajustan a ninguna de las dos situaciones anteriores. 
El modelo 1 de la Tabla 2 muestra que no existe relación estadística entre los rendimientos de mercado y el grado de control que ejercen sobre la misma sus propietarios, lo que sugiere que los administradores de aquellas empresas que enfrentan un control más directo parecen no obtener mejores resultados. Sin embargo, este resultado se alinea con la evidencia internacional, la que está lejos de ser concluyente al respecto. En efecto, Gadhoum et al. (2005) presentaron un resumen de muchos estudios con evidencia mixta, a favor y en contra de esta relación, es decir una relación desempeñoconcentración muy débil. Estimaciones adicionales usando rendimientos al cuadrado (no reportadas), no mejoraron los resultados, y de este modo, una relación no lineal puede ser descartada.

\section{Variables de control}

Para efectuar comparaciones apropiadas del desempeño de las empresas, se controla por algunas de sus características fundamentales. Para esto se han incorporado a la regresión base tres grupos de variables de control o instrumentos: medidas de riesgo de las empresas, otras características de las empresas, y un grupo de características de los principales propietarios de las mismas. Naturalmente pudiera postularse un listado mucho más extenso de variables de control, incluyendo algunas más difíciles de medir con precisión en Chile, como por ejemplo las características de la gerencia, prácticas de gobierno corporativo, tipo de propiedad y de control. Sin embargo, se cree que las variables usadas logran capturar la esencia de la relación bajo estudio.
La posible endogeneidad es evaluada a través del test de Hausman (1978), el que permite verificar la presencia de correlaciones entre la heterogeneidad no observada y las variables explicativas, permitiendo concluir si la estimación de mínimos cuadrados en dos etapas usando instrumental variables es realmente válida. Para esto se compara los estimadores de mínimos cuadrados en dos etapas de un pequeño grupo de instrumentos versus otro grupo mayor, el que contiene al primer grupo. La hipótesis nula establece exogeneidad, de modo que el rechazo de la misma sugiere el uso de las variables instrumentales propuestas.

a) Medidas de riesgo de las empresas

Puesto que empresas con mayor riesgo se espera que obtengan un mayor rendimiento (no corregido) respecto a las empresas de menor riesgo, el riesgo es una variable de control. La necesidad de corregir por riesgo es el principio en el cual se basan medidas de desempeño clásicas como las de Jensen (1968) y Treynor (1966). En este caso, para cada empresa se estiman dos medidas de riesgo:

- Beta Local $(\beta)$ : El coeficiente de riesgo sistemático o $\beta$ de las empresas, está dado por:

$$
\text { it } \frac{\text { Co var( }\left(R_{i t}, R m t\right)}{\operatorname{Var}(R m t)}
$$

(Ecuación 3)

donde $R_{i t}$ es el retorno de las acciones de la empresa i en el mes $\mathrm{t}$, y $R_{m t}$ es el retorno del mercado en el mes $t$, aproximado en Chile por los rendimientos del Índice de 
Precios Selectivo de las acciones (IPSA ${ }^{5}$ ) de la Bolsa de Comercio de Santiago. $\operatorname{Var}()$ y Covar() corresponden a la varianza y covarianza respectivamente. Las estimaciones del los coeficientes $\beta$ de cada empresa consideraron una serie de tiempo con datos mensuales para el periodo comprendido entre los años 2003-2008. Como precaución, es importante señalar que algunas de las limitaciones en la estimación del coeficiente $\beta$ pueden ser especialmente relevantes en el caso chileno, especialmente la inestabilidad de los coeficientes y a las transacciones discontinuas debido a la baja presencia (Soria y Zúñiga, 1996), entre otras.

- Corrección de Godfrey-Espinoza: Una segunda medida de riesgo, que parece superar los problemas señalados para el coeficiente $\beta$, es la propuesta de Godfrey y Espinoza (1996), quienes estiman que en países emergentes el coefi- ciente $\beta$ debe considerar una corrección en el coeficiente de correlación, debido a la imperfecta integración a los mercados internacionales. En lugar de una correlación entre $R_{m t}$ (el mercado de EEUU) y los rendimientos de las empresas locales cercana a 1.0, estiman que esta queda mejor representada $\operatorname{con} \rho=0,6$. Así, puesto que Covar $\left(R_{m t}, R_{i t}\right)$.DS $\left(R_{i t}\right)$.DS $\left(R_{m t}\right)$, con $\rho=0,6$ se tiene que:

it ajustado $0.6 \frac{D S\left(R_{i t}\right)}{D S\left(R_{m t}\right)}$ (Ecuación 4)

donde DS() representa la desviación estándar. Estimaciones recientes han mostrado que esta corrección presenta un mejor ajuste en comparación con el coeficiente $\beta$ no corregido (Zúñiga y Soria, 2009), a lo menos en el sector pesquero acuícola chileno.

El Gráfico 3 muestra el histograma para las estimaciones de los coeficientes

\section{Gráfico 3}

Coeficientes de riesgo sistemático ( $\beta$ Local) (2003-2008)

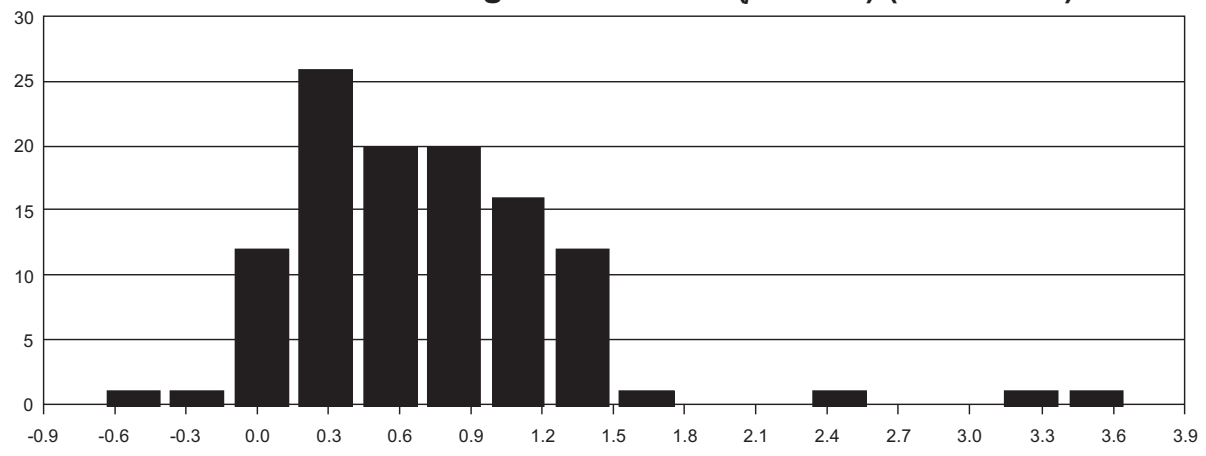

Fuente: Elaboración propia.

5 El Índice de Precio Selectivo de Acciones (IPSA) es el principal índice bursátil de Chile, elaborado por la Bolsa de Comercio de Santiago. Es un indicador de rentabilidad de las 40 acciones con mayor presencia bursátil considerando las variaciones de capital. 
de riesgo local. Se aprecia que, puesto que este coeficiente es calculado aquí respecto del IPSA, la media de este coeficiente debiera ser cercana a 1.0, lo que claramente no ocurre, centrándose más bien alrededor de 0.62. A partir de esto surge evidencia de la existencia de importantes sesgos en la estimación del $\beta$ local. Una de las razones radica en la iliquidez de muchas de las acciones locales, y también la posibilidad de que usar el IPSA como una medida de portafolio de mercado global sea demasiado incorrecto. En cualquier caso, el Gráfico 4 muestra el histograma para el coeficiente de riesgo $\beta$ ajustado, que con una media de 2.06 representa un valor mucho más plausible, si se tiene en cuenta que los rendimientos medios históricos del IPSA superan en más del doble a los rendimientos del Índice SyP500.
Los modelos 2 y 3 presentados en la Tabla 2 muestran los resultados de regresiones en que se incluyen estas medidas de riesgo adicionalmente al modelo base (modelo 1). El principal resultado es que ambas medidas de riesgo, tanto el $\beta$ local (BETA_LOCAL) como la medida de Godfrey-Espinoza (BETA_AJUST), tienen una alta capacidad explicativa del desempeño, y con signo positivo, tal como se esperaba de acuerdo a lo establecido por el CAPM $^{6}$ de Sharpe (1964). En adición, el test de Hausman sugiere la pertinencia de incluir ambas variables como parte del modelo ampliado. Estos resultados muestran que la causa de una relación no clara en el modelo 1 , no se debe a que el riesgo era una variable omitida relevante, ya que una vez capturado el efecto del riesgo sobre el desempeño sigue sin existir una relación clara desem-

\section{Gráfico 4}

Coeficientes de riesgo sistemático ajustado ( $\beta$ ajustado) 2003-2008

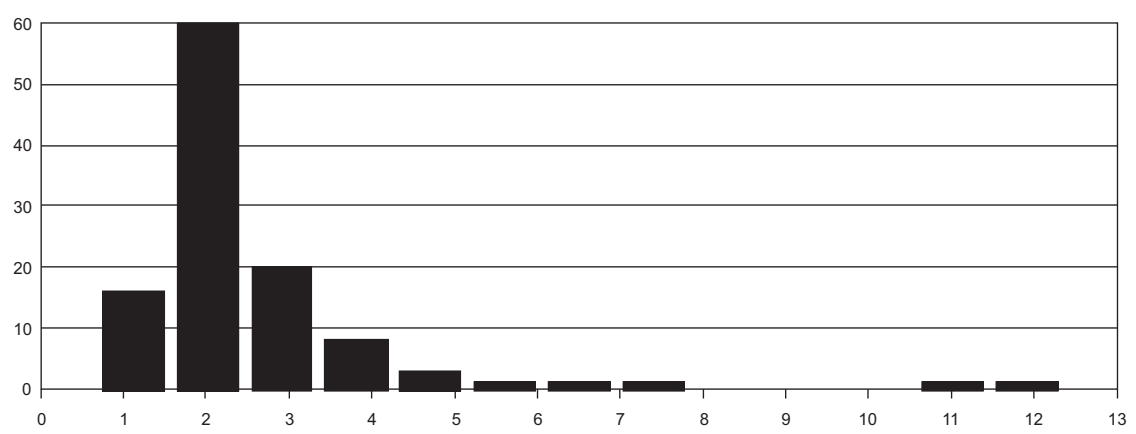

Fuente: Elaboración propia.

El CAPM, acrónimo de Capital Asset Pricing Model, o Modelo de Valoración de Activos Financieros es un modelo introducido por Jack L. Treynor, William Sharpe, John Litner y Jan Mossin independientemente, a mediados de los años 60. Posteriormente, en 1990 William Sharpe recibió el Premio Nobel de Economía por su contribución al desarrollo de este modelo. 
peño-concentración, la cual es la principal hipótesis de este estudio.

b) Características de las empresas: presencia y AFPabilidad

Es sabido que por su tamaño relativo, el mercado de valores en Chile es poco líquido (baja presencia bursátil promedio) en comparación a los promedios de los países desarrollados. En efecto, la presencia promedio en 2008 reportada por la BCS para las 112 empresas es de sólo $47.5 \%$, mientras la distribución de la liquidez tiene forma de U (Gráfico 5) reflejando una fuerte concentración en los valores extremos, e indicando que en la BCS parece existir una clara segmentación en empresas de alta presencia y de muy baja presencia, con pocos casos intermedios. Por este motivo, bajo la hipótesis de que la presencia puede contener información útil del desempeño, es incluida en el análisis.

bilidad $^{7}$ segundo lugar se evalúa siAFPaen el desempeño ajustado. La Comisión Clasificadora de Riesgo (CCR) en Chile, define el monto máximo a ser invertido en cada sociedad por parte de las Administradoras de Fondos de Pensiones (AFP). A las empresas que no logran cumplir con ciertos requisitos, se les considera de alto riesgo y se les llaman no-AFPeables. En este caso se entiende que las fuentes de generación de los recursos de la sociedad dependen en una alta proporción de empresas relacionadas, que no existe una injerencia o control significativo en la gestión de las compañías en que invierte, y además, que no existe información pública obligatoria de tales sociedades relacionadas.

\section{Gráfico 5}

\section{Presencia Bursátil en la Bolsa de Comercio de Santiago (\%) (2003-2008)}

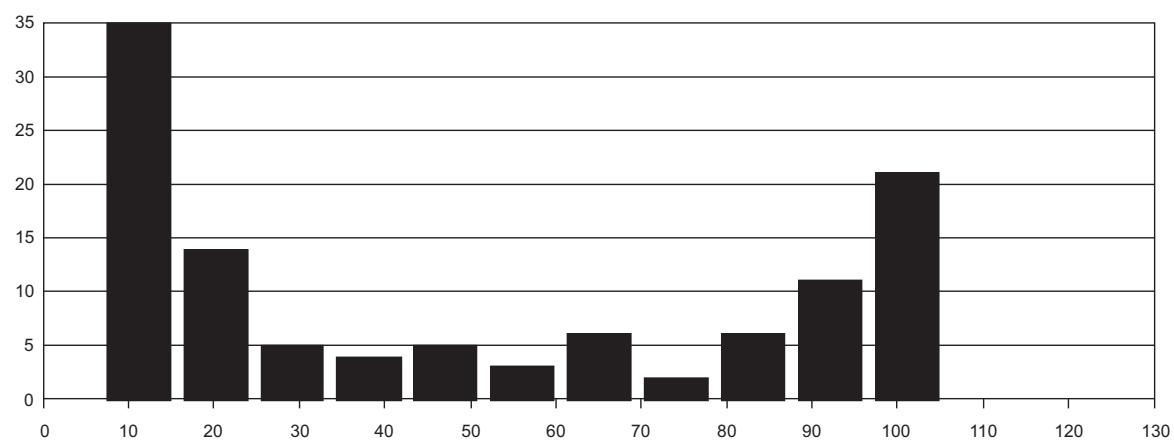

Fuente: Elaboración propia.

7 El término "Empresa AFPable" se ha hecho común en Chile para referirse a las sociedades en las cuales las Administradoras de Fondos de Pensiones están autorizadas a invertir alguna proporción de sus recursos. 
Siguiendo este razonamiento, a través de una variable Dummy se identifican las pertenecen a este grupo. La Tabla 3, las columnas (a) y (b) muestran las medias de la liquidez en bolsa y de AFPabilidad de las empresas, clasificadas en tres grupos de acuerdo a su grado de concentración. En esta tabla se calculan los promedios de las dos características de las empresas bajo análisis (presencia en bolsa y AFPabilidad) y de dos características de los principales accionistas de las empresas (si éste se encuentra inscrito en la SVS y si éste es una sociedad anónima o no). Los promedios de los valores de éstas variables se calculan para cada uno de los tres subgrupos de concentración de la propiedad: Alta, media y baja. Con el fin de determinar si existen diferencias significativas entre los grupos para cada columna se elabora un ANOVA, para el cual se muestra abajo el estadístico $F$ y la significancia de la prueba.

Se observa que una proporción importante, un $73.2 \%$, de las empresas de la analizadas son efectivamente AFPables. Un ANOVA muestra que no existen diferencias significativas a través de los tres grupos de concentración, para ninguna de las dos variables en estudio: liquidez de las acciones en Bolsa, y AFPabilidad ( $p$-value 0.4068 y 0.3483 , respectivamente). A partir de esto, se justifica suponer que dichas variables como explicativas del desempeño, en adición a la concentración de la propiedad.

Los resultados reportados al incluir estas características en el Modelo 4 y 5 (Tabla 2) muestran que ambas no logran afectar el desempeño. En efecto, el estadístico t de ambos coeficientes es claramente no significativo. En adición, el test de Hausman sugiere que no es conveniente incorporar ambas variables como explicativas del desempeño. En consecuencia, los resultados muestran que parecen no existir ventajas, desde el punto de vista de la creación de riqueza para los propietarios, en que las empresas tengan mayor liquidez, o que las AFP puedan adquirir sus acciones.

\section{Tabla 3}

Concentración según empresa y accionista mayoritario (2003-2008)

\begin{tabular}{lcccc}
\hline & $\begin{array}{c}(\mathrm{a}) \\
\text { Media de la } \\
\text { Presencia }\end{array}$ & $\begin{array}{c}\text { Media de } \\
\text { AFPabilidad }\end{array}$ & $\begin{array}{c}\text { Media de Ppal. } \\
\text { Accionista en SVS }\end{array}$ & $\begin{array}{c}\text { Media de Ppal. } \\
\text { Accionista es SA }\end{array}$ \\
\hline Concentración Alta & $35.2 \%$ & $78.6 \%$ & $42.9 \%$ & $78.6 \%$ \\
Concentración Media & $47.3 \%$ & $77.6 \%$ & $37.9 \%$ & $81.0 \%$ \\
Concentración Baja & $52.1 \%$ & $65.0 \%$ & $20.0 \%$ & $85.0 \%$ \\
Total & $47.5 \%$ & $73.2 \%$ & $32.1 \%$ & $82.1 \%$ \\
F(2,109) & 0.9069 & 1.0649 & 2.1927 & 0.1919 \\
P-value & 0.4068 & 0.3483 & 0.1165 & 0.8257 \\
\hline
\end{tabular}

Fuente: Elaboración propia. 


\section{c. Características del principal pro- pietario}

Se analiza la posible relación entre algunas características de los accionistas mayoritarios o principales propietarios de las empresas, respecto al desempeño de las mismas. Debido a que no se cuenta con información pública para caracterizar en detalle a todos los principales propietarios de las empresas estudiadas, dos son las características que se analizan: pertenencia a los registros de la SVS, y si el principal accionista es una Sociedad Anónima o no.

En primer lugar, si bien la inscripción del principal accionista en la SVS es un requisito para la emisión de títulos de oferta pública (es decir como fuente de financiamiento), este hecho debe entregar mayor transparencia de las operaciones del controlador al mercado, haciéndola más atractiva para ciertos grupos de accionistas. De acuerdo a esto, se definió una variable dummy asignando $1 \mathrm{si}$ el principal accionista de la empresa pertenece a los registros de la SVS, y 0 en otro caso. La Tabla 3, en la columna (c) muestra que en solo el $32.1 \%$ de las empresas los controladores poseen esta característica. Además, la tabla muestra que parece haber una relación positiva, aunque débil de esta característica con la concentración ( $p$-value $11,65 \%$ en el ANO-
VA), indicando una muy débil relación o tendencia a poseer más información pública del accionista mayoritario mientras mayor sea el porcentaje de propiedad que este posea.

Por otra parte, la forma de organizar legalmente las empresas puede tener una influencia en el grado de concentración y el desempeño. En efecto, la estructura tributaria-laboral de cada país empuja a los propietarios a desarrollar formas específicas de organización. En el caso de Chile, las empresas propietarias tienden a desarrollar estructuras de propiedad especialmente complicadas (por ejemplo el caso de los múltiples $\mathrm{RUT}^{8}$ ).

Algunas características notorias de las empresas propietarias en Chile merecen ser comentadas. En primer lugar, si bien en principio, los principales accionistas de las empresas pueden ser personas naturales-familias ${ }^{9}$, gobiernos, bancos, empresas no financieras y financieras, en el caso chileno solo una empresa aparece el Gobierno de Chile como principal propietario (CORFO, como propietaria principal de Zofri), y también aparecen solamente dos bancos (Banco Bilbao Vizcaya S.A. propietario principal de Bbvacl, y Citibank). También es notorio, aunque justificable desde el punto de vista tributario, el hecho de que no existan personas naturales.

8 El Rol Único Tributario (RUT) es un código único que identifica a cada empresa chilena desde el punto de vista tributario. El caso de los múltiples RUT ocurre cuando una gran empresa, que supuestamente debería operar con un único RUT, es separada en varias empresas relacionadas, cada una con un RUT distinto. Si bien parecen no existir grandes beneficios tributarios con esta figura, sí parece haberlos respecto al pago de indemnizaciones por años de servicios de los trabajadores, y con otros beneficios de tipo laboral.

9 En los Estados Unidos, cerca del $50 \%$ de todas las acciones son mantenidas por individuos, el doble que en el Reino Unido (The Economist, 2 December 1995: 107). 
Para analizar el posible impacto de la estructura organizativa del principal propietario se creó una variable dummy, con el valor 1 en el caso de que éste es una Sociedad Anónima, y 0 en otro caso. La Tabla 3, en la columna (d) muestra que en Chile, la figura más común para el propietario de las empresas en este estudio es la de sociedad anónima (mayoritariamente cerradas), siendo adoptada en un $82.1 \%$ de los casos. En la Tabla se muestra también que un ANOVA no reporta diferencias estadísticas de las características de los propietarios cuando éstos son ordenados en grupos basados en la concentración de las empresas.

Al incluir ambas variables dummy en la regresión base, los resultados reportados en el modelo 6 de la Tabla 2 muestran que no se detectó una relación estadística entre el desempeño y el reporte sistemático de información pública por parte del principal accionista. Los resultados del modelo 7 de la Tabla 2 son consistentes con los resultados previos de este estudio, en el sentido de que parecen no existir ventajas respecto a las características del principal propietario. Luego, globalmente, los resultados indican que en Chile no existe una relación entre el grado de concentración de la propiedad y el desempeño de las empresas, aún después de haber corregido por las características de los propietarios de las mismas. En adición, al igual que en el caso de los modelos 4 y 5 , el test de Hausman no sugiere incluir las características del principal propietario como explicativas del desempeño corporativo.

En síntesis, al analizar los resultados obtenidos en función del análisis teórico, los resultados parecen ser consis- tentes con los trabajos de Le Fort y Walker (1999) acerca de beneficios en la mantención de propiedad de empresas subsidiarias, el de Agosin y Pastén (2003) acerca de una discriminación moderada contra los pequeños accionistas y tenedores de bonos, el trabajo de Maquieira et al. (2003) acerca beneficios anormales de los mayoritarios en desmedro de los minoritarios, y también de Espinoza y Maquieira (2010) quienes no encuentran una relación significativa entre estructura de propiedad y desempeño.

\section{Conclusiones}

Uno de los principales resultados de este estudio sugiere que la relación entre desempeño y grado de concentración de las empresas es inexistente en Chile, esto a lo menos cuando se considera como indicador de desempeño el rendimiento de mercado del patrimonio.

Al corregir por la posibilidad de que la relación desempeño-concentración esté contaminada por la presencia de algunas variables omitidas, se incorpora en el modelo una serie de variables explicativas, que incluyen el riesgo de las empresas, ciertas características de las mismas y algunas características de los principales propietarios. Como resultado, se obtiene evidencia de que una medida de riesgo de mercado corregida de cada empresa, el coeficiente $\beta$ de Godfrey-Espinoza, logra explicar fuertemente el desempeño y con el signo esperado, un resultado consistente con el Modelo de Valoración de Activos de Capital de Sharpe.

El estudio muestra también que la AFPabilidad (como proxy contable de un bajo riesgo) y la liquidez de las empresas 
en la Bolsa de Comercio de Santiago, ambas características no logran afectar los rendimientos de mercado. Es más, tampoco logran afectar la relación desempeño-concentración de la propiedad.

Los resultados también arrojaron que los rendimientos no son sensibles al hecho de que el principal propietario posea la estructura de Sociedad Anónima, indicando que la estructura societaria del accionista mayoritario no determina el grado de concentración de las empresas que poseen. Finalmente, se concluyó que el hecho de que el principal propietario esté inscrito en la SVS tampoco tiene efecto alguno en el desempeño.

Globalmente, lo obtenido se alinea con la evidencia internacional, en el sentido de que la relación riesgo-rentabilidad positiva está bastante bien documentada a partir de inicios de los años 70 , y de que la relación desempeño-concentración de la propiedad ha mostrado una evidencia muy poco concluyente, lo que ha sido también reportado en Chile.

Por último, se debe destacar que los resultados del estudio son significativos estadística y económicamente. La robustez de los mismos ha sido evaluada en lo que se refiere a la endogeneidad a través de la prueba de Hausman. Los outliers fueron apropiadamente tratados al momento de construir la base de datos, y la posibilidad de una relación no lineal desempeño-concentración de la propiedad también fue analizada. Potenciales dificultades en los tests estadísticos relacionadas con la heterocedasticidad fueron también considerados aplicando el enfoque de errores robustos de White.
Aún falta reunir mucha evidencia empírica para explicar satisfactoriamente tales relaciones, las que incluso pueden ser no lineales, o también pueden ser verdaderas sólo para determinados empresas de ciertos tamaños, y bajo ciertos ambientes regulatorios. Como futuras líneas de investigación surge la extensión del análisis hacia otros países de la región, incorporando también economías desarrolladas. Sin embargo esto solo tiene sentido en la medida de que los estados financieros de las empresas chilenas sean confeccionados en base al estándar IFRS, de modo de asegurar un mínimo aceptable de comparabilidad intertemporal. Con esto, será posible trabajar a futuro de un modo más confiable construyendo paneles de datos.

\section{Referencias Bibliográficas}

Acharya, Viral y Bisin, Aalberto (2009). Managerial hedging, equity ownership, and firm value. RAND Journal of Economics, 40, (1), pp. 47-77.

Agosin, Manuel y Pastén, Ernesto (2003). Corporate Governance in Chile. Documentos de Trabajo, Central Bank of Chile. $\mathrm{N}^{\circ} 209$, Mayo.

Apreda, Rodolfo (2001). Corporate Governance in Argentina: the outcome of economic freedom (1991-2000). Corporate Governance: An International Review, Blackwell Publishing, vol. 9(4), pp. 298-310, October.

Bebczuk, Ricardo (2005). Corporate governance and ownership: Measurement and impact on corporate performance and dividend policy in Argentina. Working Paper 59. La Plata: Universidad Nacional de La Plata-IADB.

Becht, Marco; Bolton, Patrick y Roell A., Ailsa. (2003). Corporate governance and control, in: G.M. Constantinides y M. 
Harris y R. M. Stulz (ed.), Handbook of the Economics of Finance, edition 1, volume 1, chapter 1, pp. 1-109 Elsevier.

Berle, Adolf y Means, Gardiner (1932). The modern corporation and private property, New York: Harcourt, Brace, and World.

Bethel, Jennifer y Liebeskind, Julia (1993). The effect of ownership structure on corporate restructuring. Strategic Management Journal, Summer Special Issue 14: 15-31.

Brailsford, Tim, Barry Oliver and Lay Sandra Pua (2002). On the relation between ownership structure and capital structure. Accounting and Finance 42 (2002) pp. 1-26.

Castañeda, Gonzalo (2000). Governance of large corporations in Mexico and productivity implications. Revista ABANTE, 3 (1), pp. 57-89.

De Michele, Roberto (2000). Corporate governance: An introduction to the Argentine case. SELA Working Paper. New Haven: Yale Law School.

Espinosa, Christian y Maquieira, Carlos (2010). Desempeño y concentración de la propiedad en empresas chilenas emisoras de American Depositary Receipts (ADR). Cuad. Adm., 23(40), pp. 95-116.

Fuenzalida, Darcy, Mongrut, Samuel; Nash, Mauricio y Benavides, Julián (2008). Estructura Propietaria y Rendimientos Bursátiles en Suramérica. Cuad Adm. Bogotá (Colombia). 21 (3.1): pp. 11-35, enero-junio de 2008.

Gadhoum, Yoser; Noiseux, Marie-Hélène y Zeghal, Daniel (2005). Demystifying the Illusion of the Positive Effects of Ownership Concentration on Corporate Performance. Investment Management and Financial Innovations, 4/2005.

Gedajlovic, Eric y Shapiro, Daniel (1998). Management and ownership effects: evi- dence from five countries. Strategic Management Journal, 19: pp. 533553.

Godfrey Stephen y Espinoza, Ramón (1996). A practical approach to calculating costs of equity for investments in emerging markets. J. Appl. Corp. Financ. Fall. pp. 80-89.

Gutiérrez, Luis, Pombo, Carlos y Taborda, Rodrigo (2008). Ownership and control in Colombian Corporations. The Quarterly Review of Economics and Finance. Volume 48, pp. 22-47.

Hausman, Jerry A. (1978). Specification Tests in Econometrics. Econometrica, 46 (6), pp. 1251-1271.

Heckman, James J. (1979). Sample selection bias as a specification error. Econometrica, 47, pp. 153-61.

Hoskisson, Robert, Johnson, Richard y Moesel, Douglas (1994). Corporate divestiture intensity in restructuring firms: Effects of governance, Strategy, and Performance. Academy of Management Journal, 37(5): pp. 1207-1251.

Husted, Bryan y Serrano, Carlos (2002). Corporate governance in Mexico. Journal of Business Ethics, 37 (3), pp. 337-348.

Jensen, Michael J. (1968). The Performance of Mutual Funds in the Period 19451964. Journal of Finance, Vol. 23, No. 2 (May), pp. 389-416.

Jensen, Michael y Meckling, William (1976). Theory of the firm: Managerial behavior, agency costs, and ownership structure. Journal of Financial Economics (JFE), Vol. 3, No. 4, pp. 305360.

Le Fort, Fernando y Walker, Eduardo (1999). Ownership and capital structure of Chilean conglomerates: Facts and hypotheses for governance. Revista Abante, Vol. 3, № 1, pp. 3-27.

Majluf, Nicolás, Abarca, Nureya; Rodríguez, Darío y Fuentes, Luis (1988). Gover- 
nance and Ownership Structure In Chilean Economic Groups. Abante, Vol. 1, No 1, pp. 111-139 (abril ).

Maquieira, Carlos; Zurita, Salvador; García, Valeria y Velasco, María (2003). Valuation of Investment Companies in Chile. Estudios de Administración. Vol 10, Num 2, pp. 1-25.

McConnell, John y Servaes, Henry (1990). Additional evidence on equity ownership and corporate value. Journal of Financial Economics 27 (2), pp. 595612.

Morck, Randall; Shleifer, Andrei y Vishny, Robert (1988). Management Ownership and Market Valuation: An Empirical Analysis. Journal of Financial Economics, 20, pp. 293-315.

Morck, Randall; Nakamura, Masao y Shivdasani, Anil (2000). Banks, Ownership Structure, and Firm Value in Japan. Journal of Business, Oct., Vol. 73 Issue 4, pp. 539, 29p.

Murali, Ramaswami y Welch, Jonathan (1989). Agents, owners, control and performance. Journal of Business Finance and Accounting, Summer 1989, pp. 385-398.

Orr, Duncan; Emanuel David and Wong, Norman (2005). Board Composition and the Value of New Zealand Companies. Pacific Accounting Review Vol. 17, No. 2, December 2005.

Sharpe, William F. (1964). Capital Asset Prices - A Theory of Market Equilibrium Under Conditions of Risk. The Journal of Finance XIX (3): pp. 425-42.

Shleifer, Andrei y Vishny, Robert (1997). A Survey of Corporate Governance. The
Journal of Finance, Vol. 52, No. 2 (Jun., 1997), pp. 737-783.

Shleifer, Andrei y Vishny, Robert (1986). Large shareholders and corporate control. Journal of Political Economy, 94, pp. 461-488.

Soria, Karla y Zúñiga, Sergio (1996). Comportamiento y Estabilidad de los Betas Accionarios Chilenos. Revista CLADEA, N ${ }^{\circ} 17$, pp 27-47.

Superintendencia de Bancos e Instituciones Financieras (SBIF) (2004-2008). Base de datos de todos los Bancos chilenos, disponible en línea en http://www. www.sbif.cl.

Superintendencia de Valores y Seguros de Chile (SVS) (2004-2008). Base de datos de todas las instituciones financieras inscritas, disponible en línea en http://www.svs.cl.

The Economist (1995). Investor types, p. 107. December.

Thomsen, Steen y Pedersen, Torben (2000). Ownership structure and economic performance in the largest European countries. Strategic Management Journal, 21: pp. 689-705.

Treynor, Jack L. (1966). How to rate management investment funds. Harvard Business Review, vol 43, no. 1 (January-February): pp. 63-75.

White, Harry (1980). A HeteroskedasticityConsistent Covariance Matrix Estimator and Direct Test for Heteroskedasticity. Econometrica, Vol. 48, pp. 817838.

Zúñiga, Sergio y Soria, Karla (2009). Costo del Capital en el Sector Pesquero-Acuícola Chileno. Interciencia, Aug. 2009, Vol. 34 Nº 8 , pp. 543-550. 